\title{
On-orbit performance of the DAMPE BGO calorimeter
}

\section{Yifeng Wei, ${ }^{a, b, *}$ F.C.T. Barbato, ${ }^{c, d}$ E. Casilli, ${ }^{e, f}$ F. de Palma ${ }^{e, f}$ and G. Marsella ${ }^{e, f}$ on behalf of the DAMPE Collaboration}

(a complete list of authors can be found at the end of the proceedings)

${ }^{a}$ State Key Laboratory of Particle Detection and Electronics, University of Science and Technology of China, Hefei 230026, China

${ }^{b}$ Department of Modern Physics, University of Science and Technology of China, Hefei 230026, China

${ }^{c}$ Gran Sasso Science Institute (GSSI), Via Iacobucci 2, I-67100 L'Aquila, Italy

${ }^{d}$ Istituto Nazionale di Fisica Nucleare (INFN)-Laboratori Nazionali del Gran Sasso, I-67100 Assergi, L'Aquila, Italy

${ }^{e}$ Dipartimento di Matematica e Fisica E. De Giorgi, Università del Salento, I-73100 Lecce, Italy

${ }^{f}$ Istituto Nazionale di Fisica Nucleare (INFN)-Sezione di Lecce, I-73100 Lecce, Italy

E-mail: weiyf@ustc.edu.cn

The DArk Matter Particle Explorer (DAMPE) is the first Chinese cosmic-ray direct detection experiment. It has been operating smoothly on-orbit since its successful launch at the end of 2015. Currently, its sub-detectors and the satellite are in good working order. The DAMPE payload employs a BGO Calorimeter for energy measurements, trigger and e/p identification. The calorimeter is constructed of 308 BGO crystals, and PMTs are coupled to the crystals with optical filters to readout scintillation light. In this work, we present the status and performance of the calorimeter, including status of detector units, energy measurement, especially in $\mathrm{TeV}$ range, detector endurance, and long term performance in a duration of 65 months.

$37^{\text {th }}$ International Cosmic Ray Conference (ICRC 2021)

July 12th - 23rd, 2021

Online - Berlin, Germany

\footnotetext{
*Presenter
} 


\section{Introduction}

The DArk Matter Particle Explorer (DAMPE) [1] is a high-energy cosmic-ray and gamma-ray telescope. It was launched into a sun-synchronous orbit at an altitude of $500 \mathrm{~km}$ on December 17, 2015. DAMPE is able to extend the energy range of space-based particle detectors to tens of $\mathrm{TeV}$ region with a high energy resolution. The main objectives of DAMPE are as follows: (1) to search for a signature of dark matter particles, (2) to understand the origin, propagation, and acceleration mechanisms of high-energy cosmic rays, and (3) to study the gamma-ray emission from galactic and extragalactic sources. The DAMPE experiment has observed the unexpected feature in cosmic ray electron, proton and helium spectrum [2-4].

The DAMPE detector is composed of a Plastic Scintillator Strip Detector (PSD), a SiliconTungsten Tracker (STK), a Bismuth Germanium Oxide (BGO) calorimeter and a NeUtron Detector (NUD), as shown in Fig. 1. The charge, trajectory, and the energy of incident particles are measured using the PSD, the STK, and the BGO calorimeter, respectively. The calorimeter also identifies cosmic ray electrons from massive hadron background, and the NUD improves the electron/hadron separation.

In this paper, we present the calorimeter performance, including status of detector units, energy measurement, detector endurance, and long term performance in a duration of 65 months $(01 / 2016$ $-05 / 2021)$.

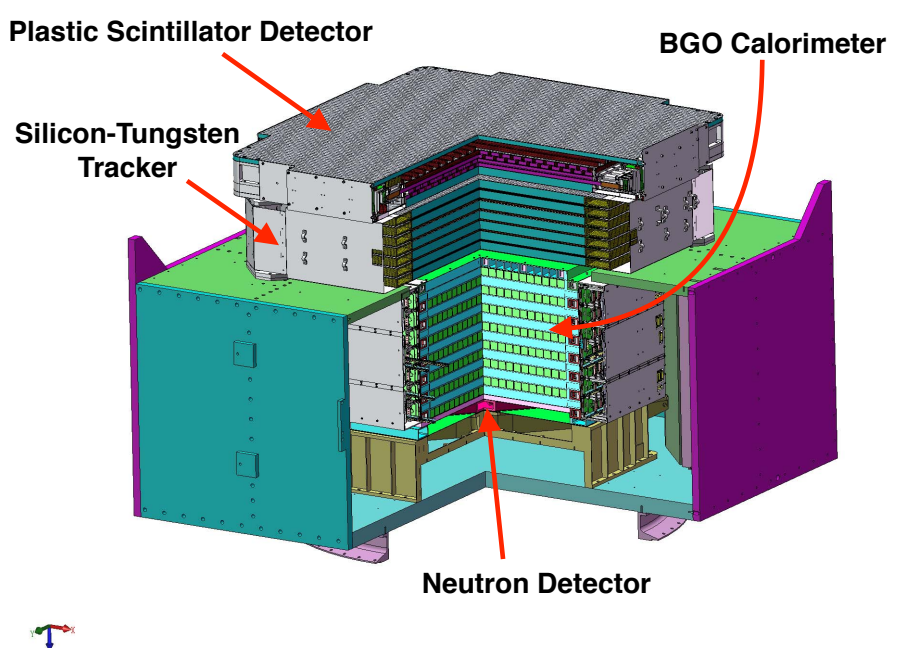

Figure 1: Schematic view of DAMPE.

\section{BGO Calorimeter}

The BGO calorimeter is the core sub-detector of DAMPE and measures the energy of incident particles with high energy resolution. The calorimeter consists of 14 layers, and each layer contains 22 BGO crystal bars with an individual size of $25 \times 25 \times 600 \mathrm{~mm}^{3}$ (Fig.2). The adjacent layers are arranged perpendicularly to reconstruct the shower topology in the calorimeter. Scintillation light is detected at both ends of a BGO crystal bar by two PMTs. The required range of energy response 
for one BGO detector unit (BGO crystal bar + PMTs) varies from $10 \mathrm{MeV}$ to $2 \mathrm{TeV}$. To obtain a wide dynamic range, a multi-dynode readout structure of the PMT base is used, with dynode 2, 5 and 8 of the PMT corresponding to low, middle and high gain, respectively. Detailed information on the readout system and electronics can be found in Ref. [1, 5-8].

The BGO calorimeter provides the trigger signal for the DAMPE data acquisition system. Until now, the trigger system works stably. The Fig. 3 shows the triggered counts per day in different energy intervals. Generally, approximately 5 million events are acquired every day, in which averagely 90 events are beyond $10 \mathrm{TeV}$.

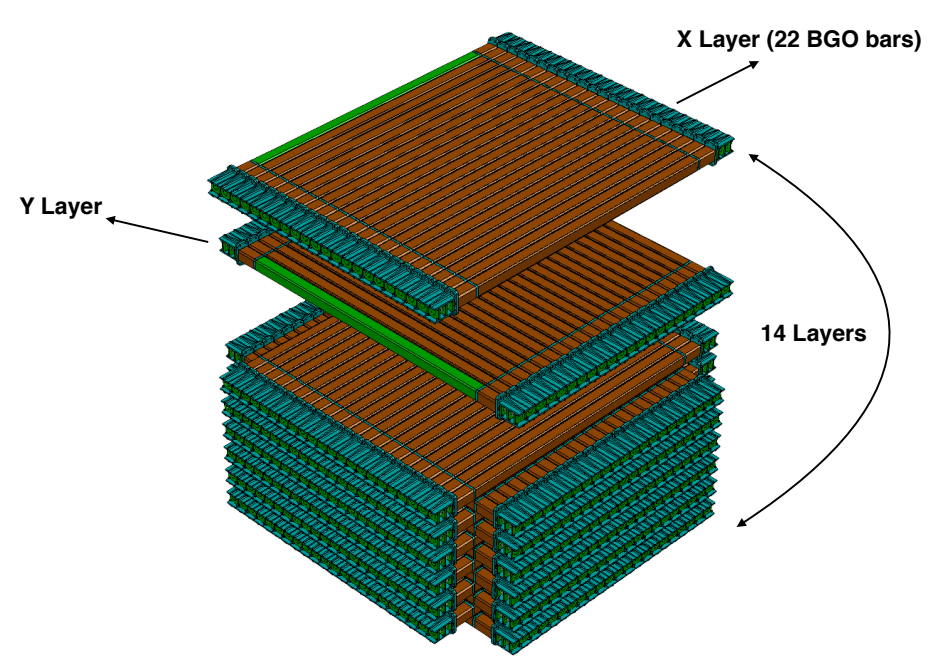

Figure 2: The BGO-crystal bar arrangement in the calorimeter. There are 14 layers, each layer containing 22 BGO crystal bars.

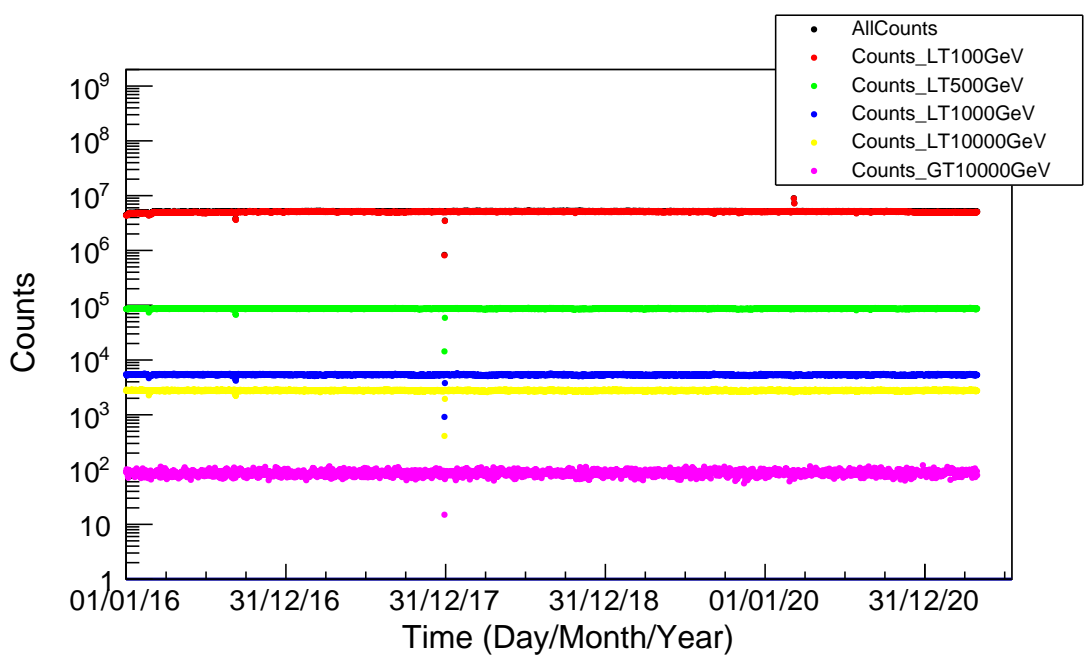

Figure 3: Triggered counts per day in different energy intervals. 


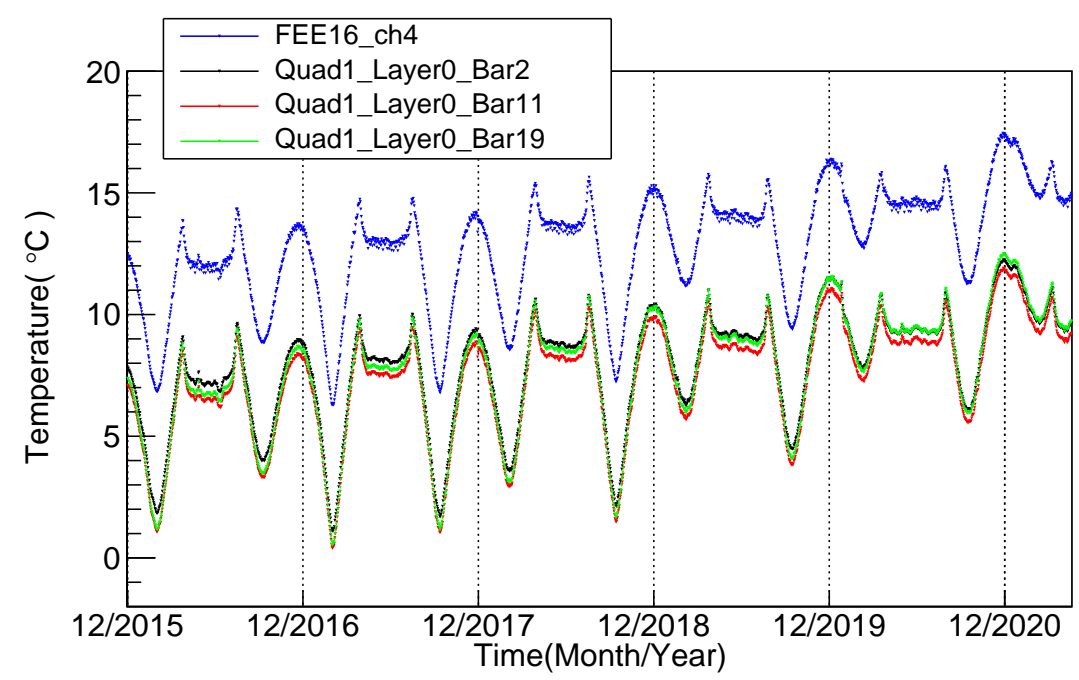

Figure 4: Temperature variations detected by four monitors equiped in the calorimeter. The blue curve represents the monitor attached on the FEE board, and the other three are those attached on the BGO crystal bars.

In orbit environment, the performance of calorimeter is affected by several factors. Temperature is one of the most important factors which depends on the angle of the satellite, as it gets heated by sunshine. Fig.4 shows temperature variations detected by four monitor equiped inside of the calorimeter. The blue curve represents the monitor that is attached on the front electronics board, and the other three are those attached on the BGO crystal bars. The temperatures varied periodically with the change of the solar illumination angle. Overall, an upward trend could be observed because of the aging of satellite thermal insulation materials.

\section{Status of Detector Units}

The calibration of individual detector unit's energy scale is crucial for whole calorimeter performance. Differ from the ground experiment, there isn't any mono-energetic sources for calibration on orbit. Cosmic-ray proton events without hadronic shower in the calorimeter, referred to as Minimum Ionizing Particle (MIP) events, are utilized to calibrated the energy scale in space. The MPV value obtained from proton MIPs spectrum is adopted as the absolute energy scale.

Fig. 5 shows the daily variation of the MPV values of the MIPs spectra and the corresponding temperature variation. Due to the temperature effect on the light yield of BGO crystals, the MPV value and the temperature are roughly anti-correlated. The temperature effect in a short time could be ignored since temperature variation rate is approximately $0.1 \%$ day. Thus, we perform MIPs calibration in every orbit to correct the temperature effect. The energy scale stablity is better than $1 \%$ after the temperature correction [5].

The calorimeter is designed to cover a wide energy dynamic range. For such a purpose, a multi-dynode read-out method is adopted. We monitor the stability of gain of dynodes. For most channels, the gains slightly increased for several percent during 65 months. A typical channel of the gain between dynode 5 and 8 is shown in Fig. 6 . 


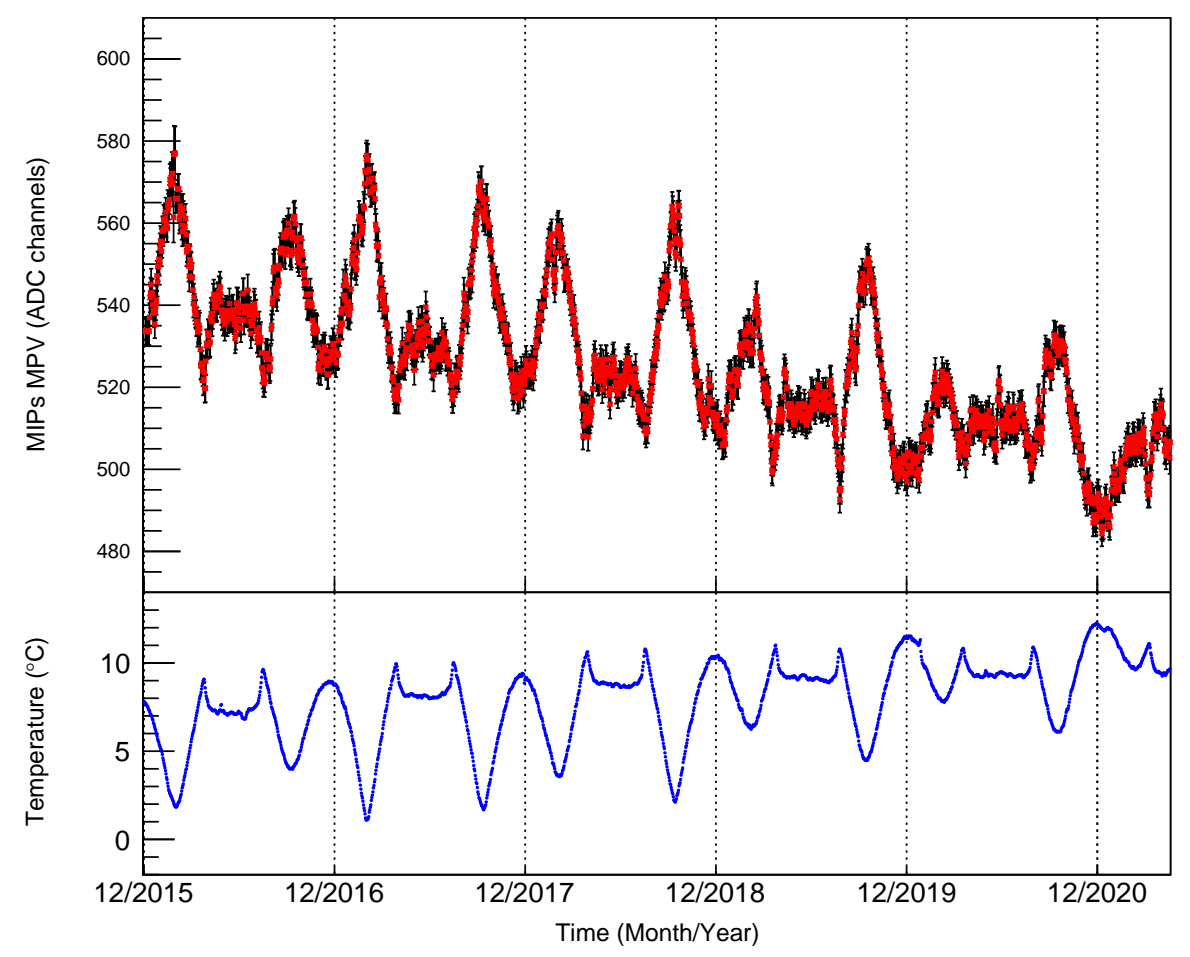

Figure 5: Variations of the MIP MPV value (upper panel) and the BGO temperature (lower panel) with time.

Due to the long-term exposure in the cosmic ray radiation environment, the BGO crystals are aged. This can lead to the decrease of fluorescence light output and deterioration of light transmittance. Thanks to the two sides readout design of a detector unit, the light attenuation length of a BGO crystal bar could be calibrated, whose mean value is $1700 \mathrm{~mm}$ [9]. We monitor the aging effect through the changing of attenuation length. Fig. 7 shows the attenuation length variation of one typical crystal with time. Averagely, the attenuation lengths of all crystals have reduced for roughly $10 \%$ in a duration of 65 months.

\section{Performance of Energy Reconstruction}

In order to evaluate the stability of energy reconstruction, we check the raw energy spectrum reconstructed in different years, as preseted in Fig. 8. In this result, the spectrum for each year show a good consistency beyond $0.5 \mathrm{GeV}$. The spectrum below $0.5 \mathrm{GeV}$ depend on the solar activity, which is different year by year. Beyond $100 \mathrm{TeV}$, it shows a cutoff in the spectrum of every year, which corresponds to the energy measurement upper limit caused by saturation of detector units. In general, the calorimeter shows good stability of energy reconstruction in past 5 years.

The TeV range, in which the community is interested, we also evaluated the energy reconstruction with cosmic-ray electrons (CREs). The energy deposition on BGO crystal located at the electromagnetic shower center is analyzed and compared with the GEANT4 Monte Carlo simulation. Fig. 9 shows the most probable value of energy deposition in shower center as a function of total energy deposition in calorimeter, where the red dots are orbit data and the open blue squares 


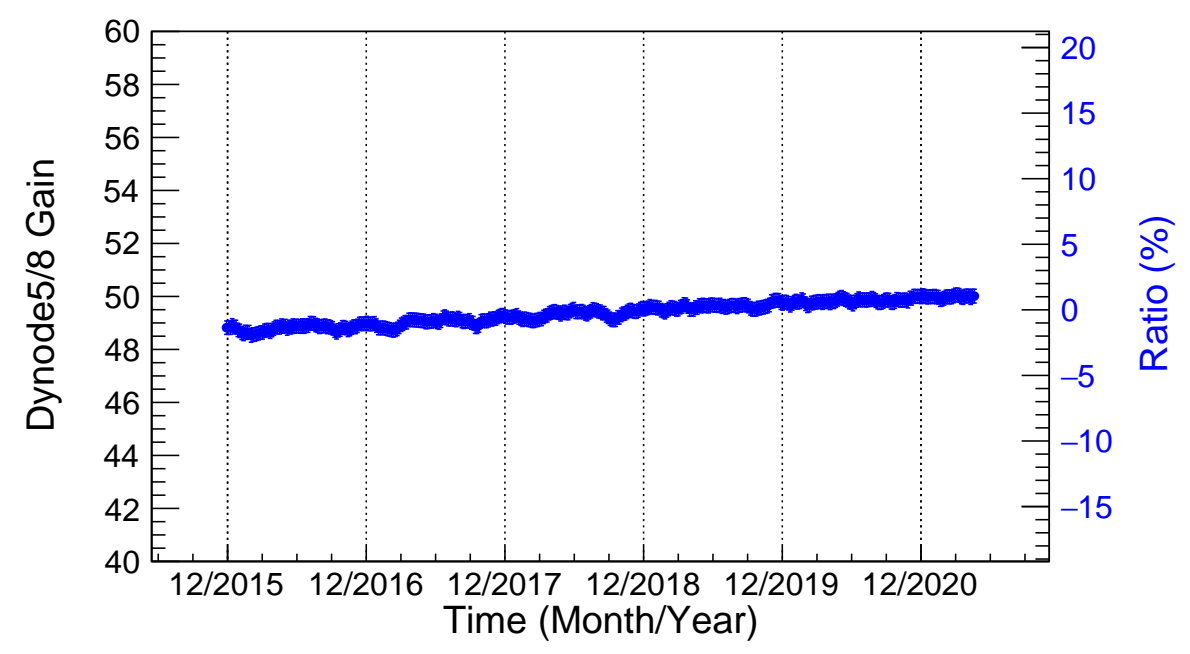

Figure 6: Variations of the PMT gain with time.

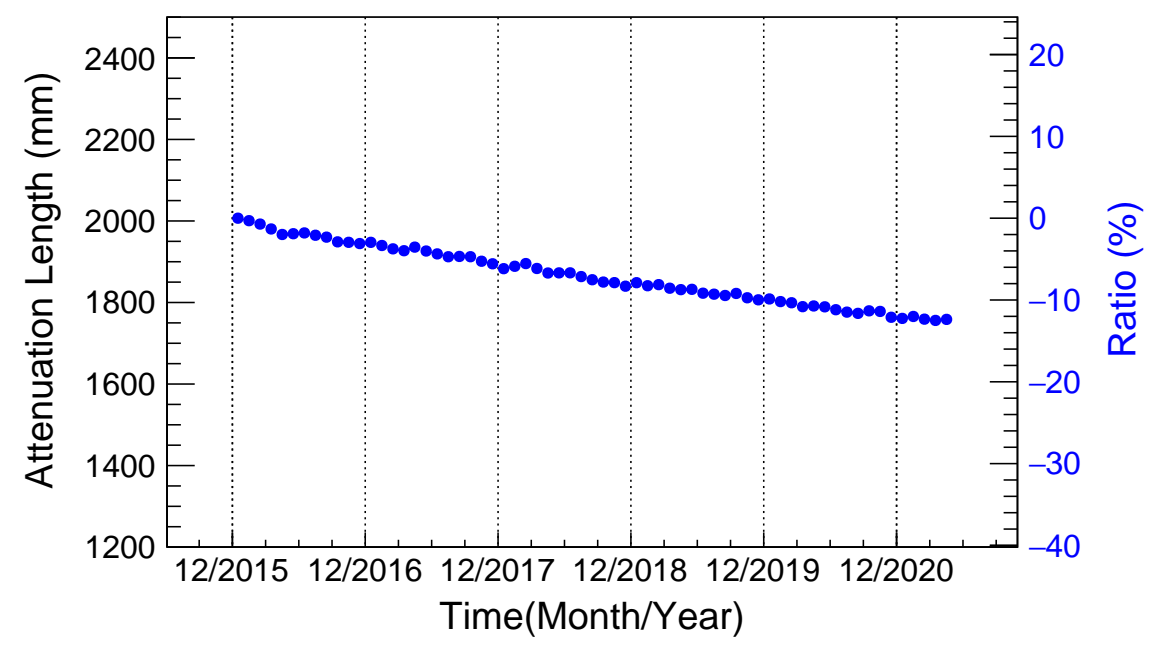

Figure 7: Variations of the BGO attenuation length with time.

are simulation. The result indicates that, up to $2.5 \mathrm{TeV}$, the energy reconstruction are consistent with the Monte Carlo one, and shows good energy linearity. The fluorescence saturation of scintillator is not observed in this result. Beyond $2.5 \mathrm{TeV}$, more statistic of electron data needs to be acquired to give a reliable validation.

\section{Summary}

The DAMPE detector has been operating nearly 5.5 years. In this work, we review the on-orbit performance of the DAMPE calorimeter. The detector units are in good working order, and proper calibration and energy reconstruction are performed to ensure high-quality data producing. 


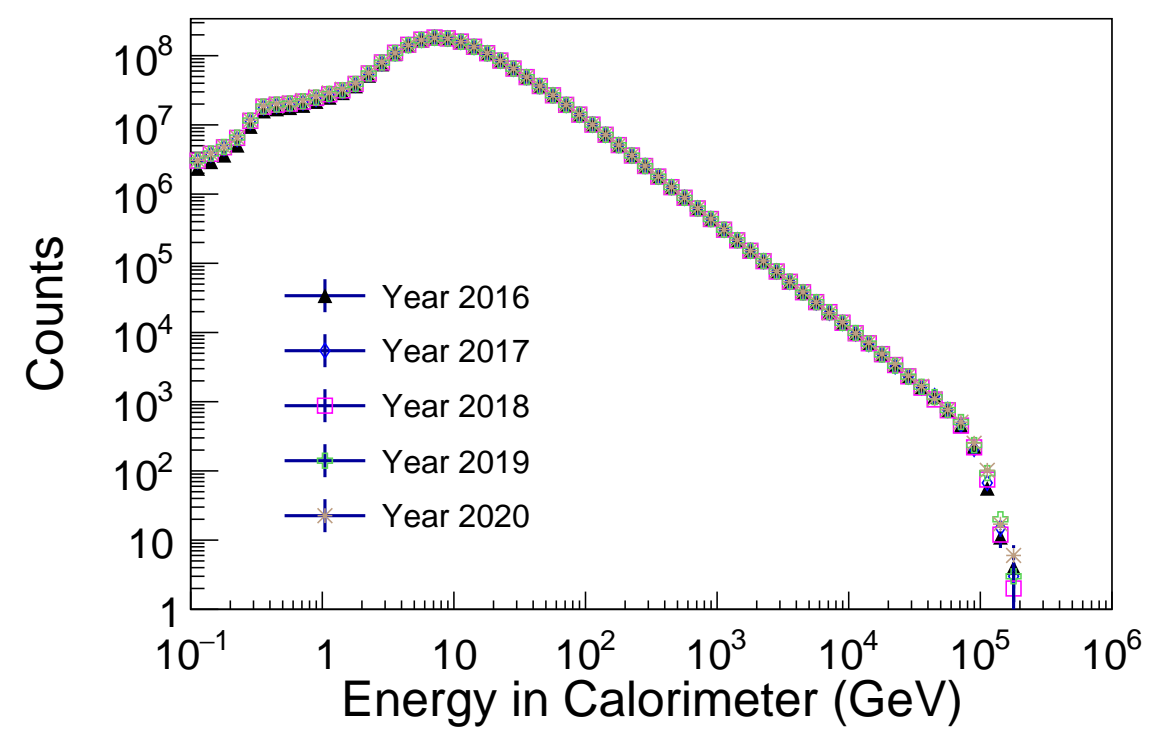

Figure 8: The deposited

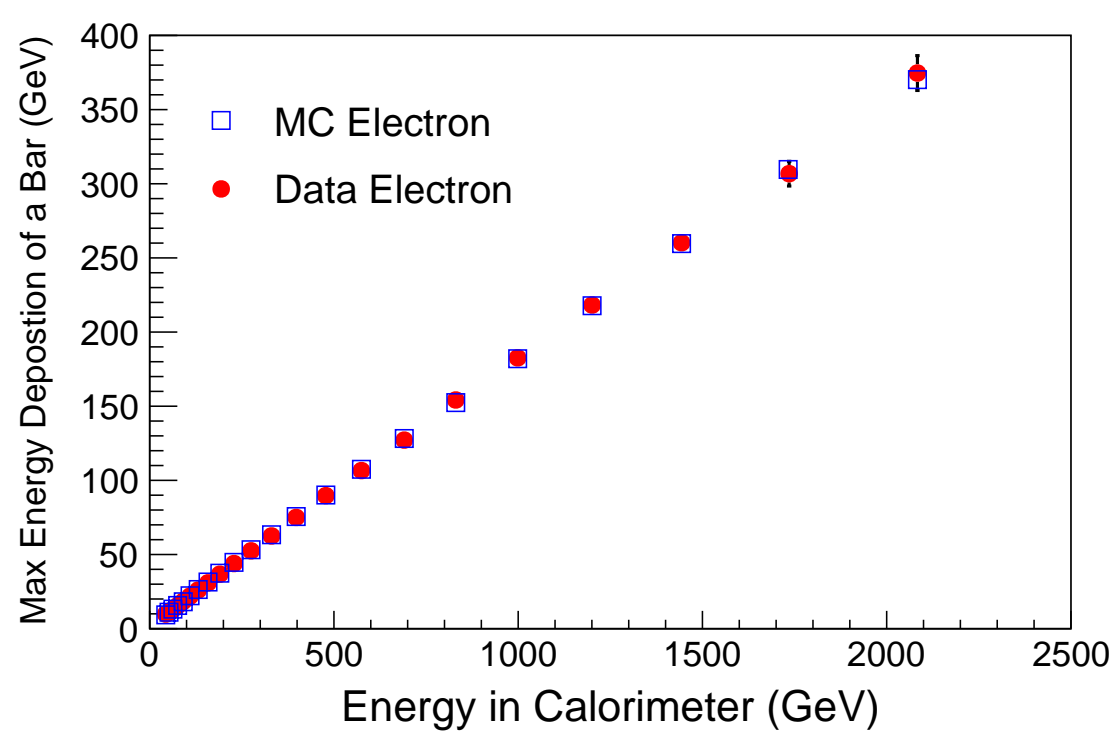

Figure 9: The deposited energy of a BGO crystal located at EM shower center as a function of the total deposited energy, where flight data are set to red dots, and MC simulations are set to open blue squares.

\section{Acknoledgement}

The DAMPE mission was funded by the strategic priority science and technology projects in space science of Chinese Academy of Sciences. In China the data analysis is supported by the National Key Research and Development Program of China (No. 2016YFA0400200), the National Natural Science Foundation of China (No. 11921003, No. 11622327, No. 12003076, No. 11722328, No. 11851305, No. U1738205, No. U1738206, No. U1738207, No. U1738208, 
No. U1738127), the strategic priority science and technology projects of Chinese Academy of Sciences (No. XDA15051100), the Young Elite Scientists Sponsorship Program by CAST (No. YESS20160196), and the Program for Innovative Talents and Entrepreneur in Jiangsu. In Europe the activities and data analysis are supported by the Swiss National Science Foundation (SNSF), Switzerland, and the National Institute for Nuclear Physics (INFN), Italy.

\section{References}

[1] J. Chang et al., (DAMPE Collaboration), The dark matter particle explorer mission, Astroparticle Physics 95 (2017) 6-24.

[2] G. Ambrosi et al., (DAMPE Collaboration), Direct detection of a break in the teraelectronvolt cosmic-ray spectrum of electrons and positrons. Nature 552.7683 (2017): 63.

[3] Q. An et al., (DAMPE Collaboration), Measurement of the cosmic ray proton spectrum from $40 \mathrm{GeV}$ to $100 \mathrm{TeV}$ with the DAMPE satellite. Science advances 5.9 (2019): eaax3793.

[4] F. Alemanno et al., (DAMPE Collaboration), Measurement of the cosmic ray helium energy spectrum from $70 \mathrm{GeV}$ to $80 \mathrm{TeV}$ with the DAMPE space mission. Physical Review Letters 126.20 (2021): 201102.

[5] G. Ambrosi et al., (DAMPE Collaboration), The on-orbit calibration of dark matter particle explorer. Astroparticle Physics 106 (2019): 18-34.

[6] Z. Y. Zhang et al., Design of a high dynamic range photomultiplier base board for the BGO ECAL of DAMPE. Nuclear Instruments and Methods in Physics Research Section A: Accelerators, Spectrometers, Detectors and Associated Equipment 780 (2015): 21-26.

[7] Y. F. Wei et al., Performance of the BGO Detector Element of the DAMPE Calorimeter. IEEE Transactions on Nuclear Science 63.2 (2016): 548-551.

[8] C. Q. Feng et al., Design of the readout electronics for the BGO calorimeter of DAMPE mission. IEEE Transactions on Nuclear Science 62.6 (2015): 3117-3125.

[9] L. B. Wu et al., Energy correction based on fluorescence attenuation of DAMPE. Research in Astronomy and Astrophysics 20.8 (2020): 118. 


\section{Full Authors List: DAMPE Collaboration}

F. Alemanno ${ }^{1,2}$, Q. An ${ }^{3,4}$, P. Azzarello ${ }^{5}$, F. C. T. Barbato ${ }^{1,2}$, P. Bernardini ${ }^{6,7}$, X. J. Bi ${ }^{8,9}$, M. S. Cai ${ }^{10,11}$, E. Casilli6, ${ }^{6,7}$ E. Catanzani ${ }^{12}$, J. Chang ${ }^{10,11}$, D. Y. Chen ${ }^{10}$, J. L. Chen ${ }^{13}$, Z. F. Chen ${ }^{10,11}$, M. Y. Cui ${ }^{10}$, T. S. Cui ${ }^{14}$, Y. X. Cui ${ }^{10,11}$, H. T. Dai ${ }^{3,4}$, A. De Benedittis ${ }^{6,7}$,

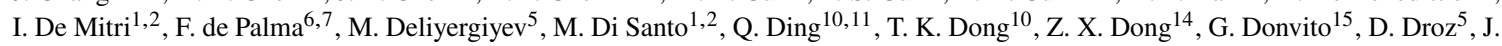
L. Duan ${ }^{13}$, K. K. Duan ${ }^{10}$, D. D’Urso ${ }^{12, *}$, R. R. Fan ${ }^{8}$, Y. Z. Fan ${ }^{10,11}$, K. Fang ${ }^{8}$, F. Fang ${ }^{13}$, C. Q. Feng ${ }^{3,4}$, L. Feng ${ }^{10}$, P. Fusco ${ }^{15,16}$, M. $\mathrm{Gao}^{8}$, F. Gargano ${ }^{15}$, K. Gong ${ }^{8}$, Y. Z. Gong ${ }^{10}$, D. Y. Guo ${ }^{8}$, J. H. Guo ${ }^{10,11}$, S. X. Han ${ }^{14}$, Y. M. Hu ${ }^{10}$, G. S. Huang ${ }^{3,4}$, X. Y. Huang ${ }^{10,11}$,

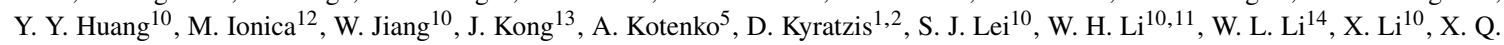

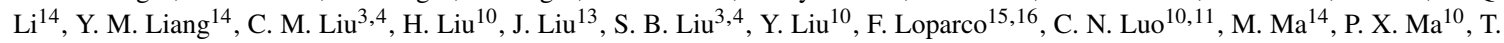
$\mathrm{Ma}^{10}$, X. Y. Ma ${ }^{14}$, G. Marsella ${ }^{6,7 \dagger}$, M. N. Mazziotta ${ }^{15}$, D. $\mathrm{Mo}^{13}$, X. Y. Niu ${ }^{13}$, X. Pan ${ }^{10,11}$, A. Parenti ${ }^{1,2}$, W. X. Peng ${ }^{8}$, X. Y. Peng ${ }^{10}$, C. Perrina $^{5,}$, R. Qiao ${ }^{8}$, J. N. Rao ${ }^{14}$, A. Ruina ${ }^{5}$, M. M. Salinas ${ }^{5}$, G. Z. Shang ${ }^{14}$, W. H. Shen ${ }^{14}$, Z. Q. Shen ${ }^{10}$, Z. T. Shen ${ }^{3,4}$, L. Silveri ${ }^{1,2}$, J. X. Song ${ }^{14}$, M. Stolpovskiy ${ }^{5}$, H. Su ${ }^{13}$, M. Su ${ }^{17}$, H. R. Sun ${ }^{3,4}$, Z. Y. Sun ${ }^{13}$, A. Surdo ${ }^{7}$, X. J. Teng ${ }^{14}$, A. Tykhonov ${ }^{5}$, H. Wang ${ }^{14}$, J. Z.

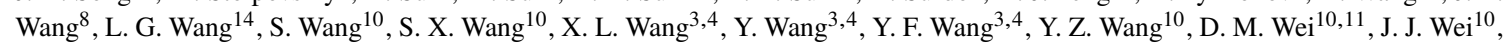
Y. F. $\mathrm{We}^{3,4}$, D. $\mathrm{Wu}^{8}$, J. Wu ${ }^{10,11}$, L. B. $\mathrm{Wu}^{1,2}$, S. S. $\mathrm{Wu}^{14}$, X. Wu $\mathrm{Wu}^{5}$ Z. Q. Xia ${ }^{10}$, H. T. Xu ${ }^{14}$, Z. H. Xu ${ }^{10,11}$, Z. L. Xu ${ }^{10}$, E. H. Xu $u^{3,4}, Z$. Z. Xu ${ }^{3,4}$, G. F. Xue ${ }^{14}$, H. B. Yang ${ }^{13}$, P. Yang ${ }^{13}$, Y. Q. Yang ${ }^{13}$, H. J. Yao ${ }^{13}$, Y. H. Yu ${ }^{13}$, G. W. Yuan ${ }^{10,11}$, Q. Yuan ${ }^{10,11}$, C. Yue ${ }^{10}$, J. J. Zang $^{10 \S}$ S. X. Zhang ${ }^{13}$, W. Z. Zhang ${ }^{14}$, Yan Zhang ${ }^{10}$, Yi Zhang ${ }^{10,11}$, Y. J. Zhang ${ }^{13}$, Y. L. Zhang ${ }^{3,4}$, Y. P. Zhang ${ }^{13}$, Y. Q. Zhang ${ }^{10}$, Z. Zhang $^{10}$, Z. Y. Zhang ${ }^{3,4}$, C. Zhao ${ }^{3,4}$, H. Y. Zhao ${ }^{13}$, X. F. Zhao ${ }^{14}$, C. Y. Zhou ${ }^{14}$, and Y. Zhu ${ }^{14}$

${ }^{1}$ Gran Sasso Science Institute (GSSI), Via Iacobucci 2, I-67100 L'Aquila, Italy

${ }^{2}$ Istituto Nazionale di Fisica Nucleare (INFN) -Laboratori Nazionali del Gran Sasso, I-67100 Assergi, L'Aquila, Italy

${ }^{3}$ State Key Laboratory of Particle Detection and Electronics, University of Science and Technology of China, Hefei 230026, China

${ }^{4}$ Department of Modern Physics, University of Science and Technology of China, Hefei 230026, China

${ }^{5}$ Department of Nuclear and Particle Physics, University of Geneva, CH-1211, Switzerland

${ }^{6}$ Dipartimento di Matematica e Fisica E. De Giorgi, Universita‘ del Salento, I-73100, Lecce, Italy

${ }^{7}$ Istituto Nazionale di Fisica Nucleare (INFN) - Sezione di Lecce, I-73100, Lecce, Italy

${ }^{8}$ Institute of High Energy Physics, Chinese Academy of Sciences, Yuquan Road 19B, Beijing 100049, China

${ }^{9}$ University of Chinese Academy of Sciences, Yuquan Road 19A, Beijing 100049, China

${ }^{10}$ Key Laboratory of Dark Matter and Space Astronomy, Purple Mountain Observatory, Chinese Academy of Sciences, Nanjing 210023, China

${ }^{11}$ School of Astronomy and Space Science, University of Science and Technology of China, Hefei 230026, China

${ }^{12}$ Istituto Nazionale di Fisica Nucleare (INFN) - Sezione di Perugia, I-06123 Perugia, Italy

${ }^{13}$ Institute of Modern Physics, Chinese Academy of Sciences, Nanchang Road 509, Lanzhou 730000, China

${ }^{14}$ National Space Science Center, Chinese Academy of Sciences, Nanertiao 1, Zhongguancun, Haidian district, Beijing 100190, China

${ }^{15}$ Istituto Nazionale di Fisica Nucleare (INFN) - Sezione di Bari, I-70125, Bari, Italy

${ }^{16}$ Dipartimento di Fisica "M. Merlin" dell'Universita“ e del Politecnico di Bari, I-70126, Bari, Italy

${ }^{17}$ Department of Physics and Laboratory for Space Research, the University of Hong Kong, Pok Fu Lam, Hong Kong SAR, China

*Now at Dipartimento di Chimica e Farmacia, Universita di Sassari, I-07100, Sassari, Italy.

$\dagger$ Now at Dipartimento di Fisica e Chimica "E. Segre“", Universita“ degli Studi di Palermo, via delle Scienze ed. 17, I-90128 Palermo, Italy.

¥Also at Institute of Physics, Ecole Polytechnique Federale de Lausanne (EPFL), CH-1015 Lausanne, Switzerland.

$\S$ Also at School of Physics and Electronic Engineering, Linyi University, Linyi 276000, China. 